\title{
De novo hemorrhagic sporadic cavernous malformation appearance after COVID-19 respiratory infection: illustrative case
}

\author{
Carmen R. Holmes, MD, ${ }^{1}$ Giuseppe Lanzino, MD, ${ }^{2}$ and Kelly D. Flemming, $\mathrm{MD}^{1}$ \\ Departments of ${ }^{1}$ Neurology and ${ }^{2}$ Neurosurgery, Mayo Clinic, Rochester, Minnesota
}

BACKGROUND Little is known about whether coronavirus disease 2019 (COVID-19) influences cavernous malformation (CM) formation or hemorrhage risk.

OBSERVATIONS The authors present the case of a 31-year-old patient who developed a hemorrhagic, de novo CM in the setting of a developmental venous anomaly within 3 months of COVID-19 respiratory disease. The authors speculate that COVID-19 disease stimulated formation of the CM through TLR4 inflammatory pathways and subsequently led to the hemorrhagic presentation because of hypercoagulability related to the disease.

LESSONS This case raises the possibility that COVID-19 may be a risk factor for de novo development of CMs in predisposed patients.

https://thejns.org/doi/abs/10.3171/CASE21543

KEYWORDS cavernous malformation; cavernous angioma; COVID-19; inflammation

Very little has been published regarding the influence of coronavirus disease 19 (COVID-19) on cavernous malformation (CM) formation and hemorrhage. A recent, small study suggests a possible increased risk of CM hemorrhage in patients who develop COVID$19 .^{1}$ To date, there have been no reported cases of de novo CM formation in the setting of COVID-19.

\section{Illustrative Case}

In the summer of 2020, our patient, a 31-year-old female, developed mild galactorrhea and was subsequently found to have mildly elevated prolactin level. A brain MRI with dedicated pituitary sequences showed no pituitary abnormality and was otherwise unremarkable with exception of a right temporal developmental venous anomaly (DVA) without an associated CM (Fig. 1 upper).

In late March 2021, the patient contracted COVID-19 and experienced symptoms of headache, extreme lethargy, and shortness of breath. After the acute illness, she has continued to experience chronic, residual symptoms, including fatigue, shortness of breath, and intermittent dizziness.

In early June 2021, the patient awoke with symptoms of tachycardia, visual illusions, depersonalization, and emotional lability that progressed over the course of 1 day. At this time, she visited a local emergency department and was diagnosed with a panic attack after a negative cardiac workup without neurological investigation. Over the of the course of the next 4 weeks, symptoms persisted, and certain shapes and patterns remained distorted, "like a funhouse." Due to persistent symptoms, she again sought medical attention and was diagnosed with a migraine. The patient ultimately sought care for the third time. At that time, the patient finally received head imaging. Noncontrast computed tomography of the head demonstrated a 12-mm intraparenchymal hemorrhage of the right temporal lobe. Subsequent magnetic resonance imaging (MRI) of the brain showed the characteristic appearance of a sporadic hemorrhagic CM with associated DVA and surrounding edema (Fig. 1 lower). She underwent 36-hour prolonged encephalogram monitoring without evidence of epileptiform discharges.

Following discharge, the patient continued to have fluctuating severe anxiety and depersonalization requiring multiple trials of psychiatric medications. Ultimately, her symptoms improved with Paxil and Seroquel. By the fall of 2021, symptoms of depersonalization and visual distortions had nearly resolved.

ABBREVIATIONS CM = cavernous malformation; COVID-19 = coronavirus disease 2019; DVA = developmental venous anomaly; MRI = magnetic resonance imaging. INCLUDE WHEN CITING Published November 8, 2021; DOI: 10.3171/CASE21543.

SUBMITTED September 20, 2021. ACCEPTED September 27, 2021.

(C) 2021 The authors, CC BY-NC-ND 4.0 (http://creativecommons.org/licenses/by-nc-nd/4.0/). 

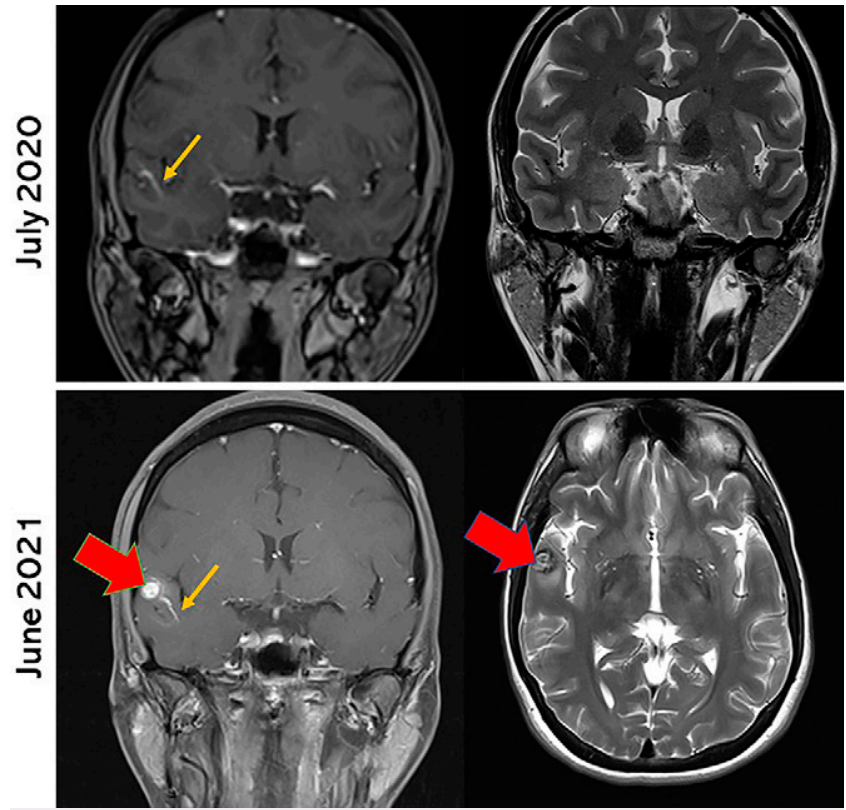

FIG. 1. Upper: Patient underwent a pituitary dedicated MRI due to mildly elevated prolactin in July of 2020. This MRI demonstrates presence of a DVA (yellow arrow) in the right temporal lobe on the T1 with gadolinium sequence but absence of a $\mathrm{CM}$ on $\mathrm{T} 2$ sequences at that time. Lower: MRI demonstrates acute hemorrhage in a right temporal CM (red arrow) on both T1 with gadolinium and T2 sequences in association with the previously seen DVA (yellow arrow).

\section{Discussion}

\section{Observations}

We report a unique case of a de novo sporadic CM appearance and hemorrhage within 2 months of COVID-19 respiratory infection. While it is well known that sporadic CMs may form in close proximity to a DVA, the time course of development and inciting events in the present case may suggest a causal link between the factors (i.e., preexisting DVA and COVID-19).

\section{Lessons}

Both basic science and clinical studies have suggested that inflammation plays a role in the formation of CM. Mice harboring familial CCM mutation exposed to gram-negative bacteria demonstrated development of CM lesions. ${ }^{2}$ In the same mice, antibiotic administration or TLR4 (Tolllike receptor 4) blockage prevented CM development. Thus, it was felt the TLR4 pathway, an important pathway activating the innate immune system, is important in the pathogenesis of CM lesions. Other supporting data include 188 patients with familial CCM, in whom single-nucleotide polymorphisms in TLR4 and CD14 were associated with increased CM lesion burden. ${ }^{3}$ In addition, clinical cohort studies suggest that patients with DVA and CM compared to those with DVA alone are more likely to have a chronic inflammatory disease ${ }^{4}$ or allergy ${ }^{5}$ in sporadic CM.

We believe COVID-19 may have caused an acute inflammatory response in this patient with a predisposing disposition (DVA), leading to the appearance of a $\mathrm{CM}$. The acute inflammatory response may have triggered the TLR4 pathway, resulting in lesion formation. It is known that the spike glycoprotein of the severe acute respiratory syndrome coronavirus 2 can bind to TLR4 receptors and are thought to play a role in the inflammation associated with the disease. ${ }^{6}$ In addition, because COVID-19 is associated with venous thrombotic events, it is plausible that the patient in question may have developed thrombosis of one of the radicles of the DVA, resulting in outflow resistance and poor $\mathrm{CM}$ drainage with resultant increased pressure in the fragile cavernous endothelial walls resulting in rupture. A recent report has also raised this possibility in patients with COVID-19. ${ }^{1}$

The MRI scan performed in the summer of 2020 was dedicated to the pituitary gland and did not contain susceptibility weighted images. It is possible that she may have had a very tiny, Zabramski type $4 \mathrm{CM}$ present, but this does not take away the fact that the aggressive behavior of her lesion occurred after the COVID-19 respiratory disease.

In conclusion, we believe this case raises questions about the possibility that de novo formation of a $\mathrm{CM}$ adjacent to a preexisting DVA may be triggered shortly (within a few weeks and not years) after a significant inflammatory response and, in particular, COVID19. Further studies may elucidate whether there are specific precautions patients with DVA may take to reduce the risk.

\section{Acknowledgments}

We thank Lea Dacy for helping with manuscript preparation.

\section{References}

1. Shkoukani A, Srinath A, Stadnik A, et al. COVID-19 in a hemorrhagic neurovascular disease, cerebral cavernous malformation. J Stroke Cerebrovasc Dis. 2021;30(11):106101.

2. Tang AT, Choi JP, Kotzin JJ, et al. Endothelial TLR4 and the microbiome drive cerebral cavernous malformations. Nature. 2017;545(7654): 305-310.

3. Choquet $\mathrm{H}$, Pawlikowska L, Nelson J, et al. Polymorphisms in inflammatory and immune response genes associated with cerebral cavernous malformation type 1 severity. Cerebrovasc Dis. 2014; 38(6):433-440.

4. Kumar S, Lanzino G, Brinjikji W, Hocquard KW, Flemming KD. Infratentorial developmental venous abnormalities and inflammation increase odds of sporadic cavernous malformation. J Stroke Cerebrovasc Dis. 2019;28(6):1662-1667.

5. Dammann P, Saban DV, Herten A, et al. Cerebral cavernous malformations: prevalence of cardiovascular comorbidities and allergic diseases compared to the normal population. Eur J Neurol. 2021; 28(6):2000-2005.

6. Kaushik D, Bhandari R, Kuhad A. TLR4 as a therapeutic target for respiratory and neurological complications of SARS-CoV-2. Expert Opin Ther Targets. 2021;25(6):491-508.

\section{Disclosures}

Dr. Lanzino reported compensation from Superior Medical Editors outside the submitted work.

\section{Author Contributions}

Conception and design: Flemming, Holmes. Acquisition of data: all authors. Analysis and interpretation of data: Flemming, Lanzino. Drafting the article: Holmes, Lanzino. Critically revising the article: Flemming, Lanzino. Reviewed submitted version of manuscript: Flemming, Lanzino. Approved the final version of the manuscript on behalf of all authors: Flemming. Administrative/technical/material support: Flemming. Study supervision: Flemming.

\section{Correspondence}

Kelly D. Flemming: Mayo Clinic, Rochester, MN. flemming.kelly@mayo.edu. 